\title{
EXISTENCE OF SOLUTIONS AND L $^{\infty}$-BOUNDS FOR QUASILINEAR DEGENERATE PARABOLIC SYSTEMS
}

\author{
D. WRZOSEK and W.M. ZAJA̧CZKOWSKI
}

Received June 6, 1998 and, in revised form, January 22, 1999

\begin{abstract}
Existence of weak solutions for systems of quasilinear degenerate parabolic equations with non-diagonal main part and nonlinear boundary conditions is proved. Under some restrictions we find also $L^{\infty}$ - bounds for the solutions.
\end{abstract}

\section{Introduction}

We consider the following quasilinear system of parabolic equations with nonlinear Neumann boundary conditions

$$
\begin{array}{lc}
\frac{\partial}{\partial t} u_{i}-\operatorname{div} \sum_{j=1}^{m} a_{i j}(x, t, u, \nabla u) \nabla u_{j}+R_{i}(x, t, u) u_{i} \\
=f_{i}(x, t, u, \nabla u) & \text { in } \Omega_{T}=\Omega \times(0, T) \\
\left.u_{i}\right|_{t=0}=u_{0 i} & \text { in } \Omega \\
\sum_{j=1}^{m} \bar{n} a_{i j} \nabla u_{j}=g_{i}(x, t, u) & \text { on } S_{T}=S \times(0, T),
\end{array}
$$

1991 Mathematics Subject Classification. 35 K; 40, 50, 55, 65 .

Key words and phrases. Quasilinear degenerate parabolic systems, existence of global solutions, $L_{\infty}$-estimates.

The paper is supported by KBN grant $N^{o} 2$ PO 3A 06508.

ISSN 1425-6908 (c) Heldermann Verlag. 
where $i=1,2, \ldots, m, \Omega \subset \mathbb{R}^{n}$ is a bounded domain with a smooth boundary $S=\partial \Omega, u=\left(u_{1}, u_{2}, \ldots, u_{m}\right) \in \mathbb{R}^{\gg}, x \in \mathbb{R}^{\ltimes}, T \in(0, \infty), \bar{n}$ is the outward unit normal vector field to $S$.

Two different kinds of matrices $\left[a_{i j}\right]_{i=1, \ldots, m}^{j=1, \ldots, m}$ are considered. In the first case we assume that

$$
a_{i j}: \Omega_{T} \times \mathbb{R}^{\gg} \times \mathbb{R}^{\gg \ltimes} \rightarrow \mathbb{R}^{\ltimes^{\not}}, \quad \beth, \beth=\nVdash, \ldots,>,
$$

satisfy the Carathéodory conditions and the strong monotonicity condition:

$$
\begin{aligned}
& \sum_{i, j=1}^{m}\left(a_{i j}\left(x, t, u, q^{\prime}\right) q_{j}^{\prime}-a_{i j}\left(x, t, u, q^{\prime \prime}\right) q_{j}^{\prime \prime}\right)\left(q_{i}^{\prime}-q_{i}^{\prime \prime}\right) \\
& \geq \underline{\alpha}\left|q^{\prime}-q^{\prime \prime}\right|^{p}, \quad p \geq 2, \quad \underline{\alpha}>0,
\end{aligned}
$$

for $u \in \mathbb{R}^{\gg}, q^{\prime}, q^{\prime \prime} \in \mathbb{R}^{\gg \times}$ a.e. in $\Omega_{T}$ and the growth condition

(H1.ii)

$$
\left|\sum_{i, j=1}^{m} a_{i j}(\cdot, u, q) q_{j}\right| \leq \bar{\alpha}|q|^{p-1}
$$

where $\bar{\alpha}$ is a positive constant.

In the second case we assume that $\left[a_{i j}\right]_{i=1, \ldots, m}^{j=1, \ldots, m}$ is an upper triangular matrix

$$
\begin{aligned}
& a_{i j} \equiv 0 \quad \text { for } \quad j<i \\
& a_{i i}: \Omega_{T} \times \mathbb{R}^{\gg} \times \mathbb{R}^{\ltimes} \rightarrow \mathbb{R}^{\ltimes^{\nvdash}}, \quad \beth=\nVdash, \ldots,>, \\
& a_{i j}: \Omega_{T} \times \mathbb{R}^{(\gg-\beth)} \times \mathbb{R}^{(\gg-\beth) \ltimes} \rightarrow \mathbb{R}^{\ltimes^{\sharp}}, \quad>\geq \beth>\beth \geq \nVdash
\end{aligned}
$$

and only its diagonal part satisfies the following monotonicity condition

$$
\begin{aligned}
(\mathbf{H 1} \mathbf{i})^{\prime} & \sum_{i=1}^{m}\left(a_{i i}\left(\cdot, u, q_{i}^{\prime}\right) q_{i}^{\prime}-a_{i i}\left(\cdot, u, q_{i}^{\prime \prime}\right) q_{i}^{\prime \prime}\right)\left(q_{i}^{\prime}-q_{i}^{\prime \prime}\right) \\
& \geq \underline{\alpha}\left|q^{\prime}-q^{\prime \prime}\right|^{p}, \quad p \geq 2, \underline{\alpha}>0,
\end{aligned}
$$

and the growth condition

$$
\left|a_{i i}\left(\cdot, u, q_{i}\right) q_{i}\right|+\left|\sum_{j>i} a_{i j}(\cdot, \bar{u}, \bar{q}) q_{j}\right| \leq \bar{\alpha} \sum_{j \geq i}\left(\left|q_{j}\right|^{p-1}+\left|u_{j}\right|^{r}+1\right) .
$$

a.e. in $\Omega_{T}$ for each $1 \leq i \leq m$, where $\bar{u} \in \mathbb{R}^{\gg-\beth}, \bar{q}=\left(q_{i+1}, \ldots, q_{m}\right), q_{i} \in$ $\mathbb{R}^{\ltimes}, r \leq p_{0}(1-1 / p), \bar{\alpha}, \underline{\alpha}$ are positive constants and $p_{0}$ is defined in (H2). From now on the hypotheses related to the second case will be denoted by prime characters.

Moreover,

$$
R_{i}: \Omega_{T} \times \mathbb{R}^{\gg} \rightarrow \mathbb{R}, \quad \beth=\nVdash, \not \models, \ldots,>
$$


satisfy the Carathéodory conditions and for $p_{0} \geq 2$

$$
\begin{aligned}
& \sum_{i=1}^{m} R_{i}(x, t, u) \leq \bar{\beta}|u|^{p_{0}-2} \quad \text { a.e. in } \quad \Omega_{T}, \\
& \underline{\beta}|u-v|^{p_{0}} \leq \sum_{i=1}^{m}\left(R_{i}(\cdot, u) u_{i}-R_{i}(\cdot, v) v_{i}\right)\left(u_{i}-v_{i}\right),
\end{aligned}
$$

where $\beta, \bar{\beta}$ are positive constants and $u, v \in \mathbb{R}^{\gg}$. In the second case we assume that $R_{i}$ can be split

$$
R_{i}(\cdot, u)=R_{i}^{I}\left(\cdot, u_{i}\right)+R_{i}^{I I}\left(\cdot, u_{i+1}, \ldots, u_{m}\right),
$$

where $R_{i}^{I}: \Omega_{T} \times \mathbb{R} \rightarrow \mathbb{R}$ and for $i>m \quad R_{i}^{I I}: \Omega_{T} \times \mathbb{R}^{\gg-\beth} \rightarrow \mathbb{R}$ and $R_{m}^{I I}=0$. Assume also that a.e. in $\Omega_{T}$

$$
\begin{array}{ll}
\text { (H2.i })^{\prime} & \left\{\begin{array}{l}
\left(R_{i}^{I}(\cdot, u) u-R_{i}^{I}(\cdot, v) v\right)(u-v) \geq \underline{\beta}(u-v)^{p_{0}}, \\
R_{i}^{I}(u) \leq \bar{\beta}|u|^{p_{0}-2} ;
\end{array} u, v \in \mathbb{R}\right. \\
\text { (H2.ii })^{\prime} & \mid R_{i}^{I I}(\cdot, w) \leq \bar{\beta}\left(1+|w|^{p_{0}-2}\right), \quad w \in \mathbb{R}^{\gg-\beth,} \\
\text { (H3) } & g_{i}: S_{T} \times \mathbb{R}^{\gg} \rightarrow \mathbb{R}, \quad \beth=\nVdash, \not \models, \ldots, \gg,
\end{array}
$$

satisfy the Carathéodory condition and

$$
\left|g_{i}(\cdot, u)\right| \leq \gamma\left(1+|u|^{b}\right), \quad \text { for } u \in \mathbb{R}^{>}, \quad \text { a.e. on } \quad \mathbb{S}_{\mathbb{T}}, \quad \gamma \geq \nvdash,
$$

where

$$
\begin{array}{lll}
b+1 \leq \min \left\{p_{0}, p\right\} & \text { if } \quad p_{0}>p \geq 2 \quad \text { or } \quad p_{0}=2 \quad \text { and } \\
b+1<p_{0} & \text { if } \quad 2<p_{0} \leq p .
\end{array}
$$

$$
f_{i}: \Omega_{T} \times \mathbb{R}^{\gg} \times \mathbb{R}^{\gg \ltimes} \rightarrow \mathbb{R}, \quad \beth=\nVdash, \not \models, \ldots,>\text { a.e. in } \Omega_{\mathbb{T}}
$$

and satisfy the Carathéodory condition and the growth condition

$$
\left|f_{i}(x, t, u, q)\right| \leq \delta\left(1+|u|^{\mu}+|q|^{\nu}\right) \quad i=1,2, \ldots, m,
$$

where $\delta, \mu, \nu \geq 0$ and

$$
\begin{aligned}
& \mu+1<p_{0} \quad \text { if } \quad p_{0}>2 \quad \text { and } \quad \mu=1 \quad \text { if } \quad p_{0}=2 \\
& \frac{1}{p_{0}}+\frac{\nu}{p}<1 \quad \text { if } \quad p_{0}>2 \quad \text { and } \quad \nu \leq \frac{p}{2} \quad \text { if } \quad p_{0}=2 .
\end{aligned}
$$

Moreover, we assume that

$$
u_{0 i} \in L^{2}(\Omega), \quad i=1,2, \ldots, m .
$$

For measurable set $A \subset \mathbb{R}^{\ltimes},|A|$ denotes its Lebesque measure.

We shall consider two problems related to the system (1.1) with the initial and boundary conditions (1.2) and (1.5) : 
(P1) — determined by the hypothesis: (H0), (H1), (H2), (H3), (H4) and

(P2) — determined by: $(\mathbf{H 0})^{\prime},(\mathbf{H 1})^{\prime},(\mathbf{H 2})^{\prime},(\mathbf{H} 3),(\mathbf{H} 4)$.

Below, we present two examples of P.D.E. systems related to $(\mathbf{P 1})$ and (P2) respectively:

Example 1. The following system of equations is quasilinear, non-diagonal and non-degenerate

$\frac{\partial u_{1}}{\partial t}-\operatorname{div}\left\{\left(a_{1}\left|\nabla u_{1}\right|^{p-2}+b_{1}\right) \nabla u_{1}+b_{2} \nabla u_{2}\right\}+a\left|u_{1}\right|^{p_{0}-2} u_{1}=f_{1}(u, \nabla u)$,

$\frac{\partial u_{2}}{\partial t}-\operatorname{div}\left\{\left(b_{3} \nabla u_{1}+\left(a_{2}\left|\nabla u_{2}\right|^{p-2}+b_{4}\right) \nabla u_{2}\right\}+b\left|u_{2}\right|^{p_{0}-2} u_{2}=f_{2}(u, \nabla u)\right.$,

where $u=\left(u_{1}, u_{2}\right)$ and $u(\cdot, 0)=u_{0}(\cdot)$,

$$
\begin{array}{ll}
\left(a_{1}\left|\nabla u_{1}\right|^{p_{0}-2}+b_{1}\right) \nabla u_{1} \cdot \bar{n}+b_{2} \nabla u_{2} \cdot \bar{n}=\gamma_{1}|u|^{b_{0}} & \text { on } S_{T}, \\
b_{3} \nabla u_{1} \cdot \bar{n}+\left(a_{2}\left|\nabla u_{2}\right|^{p-2}+b_{4}\right) \nabla u_{2} \cdot \bar{n}=\gamma_{2}|u|^{b_{0}} & \text { on } S_{T},
\end{array}
$$

where $p \geq 2, a_{i}, b_{j}, i=1,2, j=1, \ldots, 4$ and $b_{0}$ are positive constants such that

$$
\min \left\{b_{1}, b_{4}\right\}>\frac{1}{2}\left(\sqrt{\left|b_{2}\right|}+\sqrt{\left|b_{3}\right|}\right) .
$$

and $f_{1}, f_{2}$ satisfy $(\mathbf{H} 4)$ and (1.3), (1.4).

Example 2. The following triangular system is related to the class of models describing the cross-diffusion effect

$$
\begin{aligned}
& \frac{\partial u_{1}}{\partial t}-\operatorname{div}\left\{a_{1}\left|\nabla u_{1}\right|^{p-2} \nabla u_{1}+a_{2} u_{1}^{r}\left|\nabla u_{2}\right|^{\varrho-2} \nabla u_{2}\right\}=f_{1}\left(u, \nabla u_{1}, \nabla u_{2}\right), \\
& \frac{\partial u_{2}}{\partial t}-\operatorname{div}\left\{a_{3}\left|\nabla u_{2}\right|^{p-2} \nabla u_{2}\right\}=f_{2}\left(u, \nabla u_{1}, \nabla u_{2}\right), \\
& \quad u_{i}(\cdot, 0)=u_{0 i}(\cdot), \quad i=1,2 \quad \text { and } \\
& \left\{a_{1}\left|\nabla u_{1}\right|^{p-2} \nabla u_{1}+a_{2} u_{1}^{r}\left|\nabla u_{2}\right|^{\varrho-2} \nabla u_{2}\right\} \cdot \bar{n}=g_{1}(u), \\
& a_{3}\left|\nabla u_{2}\right|^{p-2} \nabla u_{2} \cdot \bar{n}=g_{2}(u),
\end{aligned}
$$

where $a_{1}, a_{3}>0, a_{2} \in \mathbb{R}$ are constants, $p, \varrho \geq 2, \quad(r / 2)+(\varrho / p) \leq 1$ with $f_{1}, f_{2}$ satisfying $(\mathbf{H} 4)$ and satisfying $g_{i}(\mathbf{H 3})$.

In the following example we demonstrate degenerate nondiagonal system for which we are not able to show existence of solutions, because the monotonicity condition is not satisfied. Nevertheless, we are able to show a priori $L^{\infty}$ bounds for such systems (see Section 3 ). 


\section{Example 3.}

$$
u_{i t}-\operatorname{div}\left(\sum_{i} a_{i j} \nabla u_{j}\right)=f_{i}(x, \nabla u) \quad i=1,2,
$$

where $a_{i i}=\alpha_{i}\left(x, t, \nabla u_{i}\right) \cdot i d, i=1,2, \alpha_{i}: \Omega_{T} \times \mathbb{R}^{\ltimes} \rightarrow \mathbb{R}$ and $a_{12}=$ $\beta_{1}|\nabla u|^{p_{1}-2} \cdot i d, a_{21}=\beta_{2}|\nabla u|^{p_{2}-2} \cdot i d, p_{1}<p, p_{2}<p$. Assume that

$$
\alpha_{i}\left(x, t, q_{i}\right) \geq \alpha\left|q_{i}\right|^{p-2}-\phi_{0}(x, t), \quad \alpha>0 .
$$

It easy to chack that for each $\varepsilon>0$

$$
\left|a_{i j} q_{i} q_{j}\right| \leq \varepsilon|q|^{p}+C_{\varepsilon}, \quad i \neq j .
$$

Hence,

$$
\sum_{i, j=1}^{2} a_{i j}(q) q_{i} q_{j} \geq \frac{\alpha}{2}|q|^{p}-\phi_{1}(x, t),
$$

where $\phi_{1}$ and $\phi_{2}$ are nonnegative measurable functions (see Proposition 3.4 for farther assumptions on the data).

Now, we introduce some spaces appearing naturally in the weak formulation of problems (P1) and (P2).

By $\|\cdot\|_{B}$ we denote a norm in a Banach space $B$ and by $\|\cdot\|_{p}$ the norm in the space $L^{p}(\Omega)$. By $W^{1, p}(\Omega), p>1$, we denote the Sobolev space equipped with the norm

$$
\|u\|_{W^{1, p}(\Omega)}=\left(\int_{\Omega}|\nabla u|^{p}+\int_{\Omega}|u|^{p}\right)^{1 / p} .
$$

$(\cdot, \cdot)$ - denotes the scalar product in the space $L^{2}(\Omega)$.

Let us also introduce

$$
\begin{aligned}
& X=L^{p}\left(0, T ; W^{1, p}(\Omega)\right), \\
& Y=L^{p_{0}}\left(0, T ; L^{p_{0}}(\Omega)\right), \\
& H=L^{2}\left(0, T ; L^{2}(\Omega)\right) .
\end{aligned}
$$

Then $X \cap Y$ is the Banach space with the norm

$$
\|u\|_{X \cap Y}=\|u\|_{X}+\|u\|_{Y}
$$

for $u \in X \cap Y$. The dual space $(X \cap Y)^{\prime}=X^{\prime}+Y^{\prime}$ is equipped with the norm

$$
\|v\|_{X^{\prime}+Y^{\prime}}=\inf _{v_{1} \in X^{\prime}, v_{2} \in Y^{\prime}} \max \left(\left\|v_{1}\right\|_{X^{\prime}},\left\|v_{2}\right\|_{Y^{\prime}}\right) \text { such that } v_{1}+v_{2}=v .
$$

Identifying $H$ with its dual, we have

$$
X \cap Y \subset H \subset X^{\prime}+Y^{\prime}
$$


with dense and continuous embeddings. Therefore, the dual pairing between the spaces $X \cap Y$ and $X^{\prime}+Y^{\prime}$ may be introduced by means of the scalar product in $H$ :

$$
(u, v)_{H}=\int_{0}^{T}(u(s), v(s)) d s .
$$

Let

$$
W=\left\{v: v \in X \cap Y, v^{\prime}=X^{\prime}+Y^{\prime}\right\}
$$

where $v^{\prime}$ is the time derivative in the sense of $X^{\prime}+Y^{\prime}$ valued distributions (see $[6])$ and

$$
\|v\|_{W}=\|v\|_{X \cap Y}+\left\|v^{\prime}\right\|_{X^{\prime}+Y^{\prime}} .
$$

We will make use of the following multiplicative inequality (see e.g. [2, 3])

$$
\begin{aligned}
& \iint_{\Omega_{T}}|v(x, t)|^{q} d x d t \\
& \leq C_{1} \iint_{\Omega_{T}}|\nabla v(x, t)|^{p} d x d t\left(\text { ess } \sup _{0<t<T} \int_{\Omega}|v(x, t)|^{2} d x\right)^{p / n},
\end{aligned}
$$

where $q=p(n+2) / n$ and $C_{i}, i=1,2,3$, here and below are positive constants. Equation (1.7) holds for functions $v \in V^{2, p}\left(\Omega_{T}\right)$ such that

$$
\frac{1}{|\Omega|} \int_{\Omega} v(x, t) d x=0 \quad \text { for a.e. } t \in(0, T)
$$

and $V^{2, p}\left(\Omega_{T}\right)$ is a Banach space with the norm

$$
\|u\|_{V^{2, p}\left(\Omega_{T}\right)}=\operatorname{ess} \sup _{0<t<T}\|u\|_{L^{2}(\Omega)}+\|\nabla u\|_{L^{p}\left(\Omega_{T}\right)} .
$$

From Ch. 1 of [3] we recall also the inequality

$$
\|u\|_{L^{q}\left(\Omega_{T}\right)} \leq C_{2}\|u\|_{V^{2, p}\left(\Omega_{T}\right)}, \quad q=p \frac{n+2}{n} .
$$

Moreover, we need the property of the trace operator

$$
\|u\|_{L^{q_{1}(S)}} \leq C_{3}\|u\|_{W^{1, p}(\Omega)},
$$

where $q_{1} \in[1,(n-1) p /(n-p)]$ for $p \in(1, n)$ and $q_{1} \in[1, \infty)$ for $p=n$.

Definition 1.1. By a weak solution of initial boundary value problem (1.1)-(1.2) and (1.5) we mean functions $u_{i} \in W, i=1,2, \ldots, m$, satisfying the following system of integral identities:

$$
\begin{aligned}
& \left(u_{i}^{\prime}, \phi_{i}\right)_{H}+\iint_{\Omega_{T}} \sum_{j=1}^{m} a_{i j}(\cdot, u, \nabla u) \nabla u_{j} \nabla \phi_{i} d x d t \\
& +\iint_{\Omega_{T}} R_{i}(\cdot, u) u_{i} \phi_{i} d x d t=\iint_{\Omega_{T}} f_{i}(\cdot, u, \nabla u) \phi_{i} d x d t
\end{aligned}
$$




$$
+\iint_{S_{T}} g_{i}(\cdot, u) \phi_{i} d S d t, \quad i=1,2, \ldots, m
$$

for each $\varphi_{i} \in X \cap Y, i=1,2, \ldots, m$ and $u_{i}(\cdot, 0)=u_{0 i}(\cdot)$.

Remark 1. Note that due to Proposition 2.1. in Section 2, $W \subset C(0, T$; $\left.L^{2}(\Omega)\right)$ and the initial condition is well defined.

We emphasize that this paper deals only with global in time solutions. The existence of local in time solutions can be proved by means of various interpolation inequalities (see [5]) for wider range of parameters $b, \mu$ and $\nu$ than that in (H3), (1.3) and (1.4). However, this topic exceeds the scope of this paper.

In Section 2 we show the existence of weak solutions for problems (P1) and (P2) and under some additional assumptions in Section 3 we prove also $L^{\infty}$ - bounds for the solutions.

As far as the existence of solutions is concerned the paper refers to the series of papers [1], [5], [9] and [10]. Although we assume parabolic term $(b(u))_{t}$ with $b=i d$, we partially generalize these papers by assuming nonlinear boundary condition and studying triangular systems.

We also generalize the existence results of [8] from the scalar case to some systems of equations. Notice also that we do not assume any monotonicity condition on nonlinear functions $f_{i}=f_{i}(x, t, u, \nabla u)$.

The structure of the system is also enriched by functions $R(u) u_{i}$ on the left hand sides which satisfy the growth conditions independently of $f_{i}$. Introducing them we want to investigate their influence on $L^{\infty}$ - bounds of solutions.

It is worth pointing out that the results of DiBenedetto [2] on the regularity of solutions of degenerate parabolic systems cannot be applied in our case since they essentially relay on the hypothesis that $\left[a_{i j}\right]$ is diagonal e.g. $a_{i j}=0$ for $i \neq j$ and

$$
a_{i i}=a|\nabla u|^{p-2} \nabla u_{i}, \quad a>0 .
$$

We extend the method of De Giorgi [4] (generalized in [8] and [2]) from a scalar case to some nondiagonal systems of equations. We do not make any assumptions on smoothness of nonlinear boundary data as it is done in $[2]$.

Notice also that the case of Dirichlet boundary conditions for a similar class of equations is studied in [10].

Finally, we describe our methods and results. To prove the existence of solutions to problems (P1), (P2) we use Faedo-Galerkin, monotonicity and compactness methods from [8, Ch. 5,6].

In view of (2.10) the existence of the approximate solution in $[0, T]$, with arbitrary $T$, follows from the Caratheodory theory of differential equations 
(see e.g. [7]). To pass to the limit with the approximate solutions we need the monotonicity conditions $\left(\right.$ H1.i), (H2.ii) for $(\mathbf{P 1})$ and $(\mathbf{H 1 . i})^{\prime},(\mathbf{H} 2 . \mathbf{i i})$ for (P2). Since the monotonicity condition for $(\mathbf{P 1})$ is very restrictive for systems we consider separately the case of the triangular system (see (P2)) for which the condidtion holds only for diagonal elements. It is worth pointing out here that the growth condition imposed on the non-diagonal terms may be the same as that on the diagonal ones.

To obtain the a priori $L^{\infty}$-estimate we apply the truncation method of DeGiorgi for the scalar case developed by DiBenedetto in [2, Ch. 5] by using the test function $\phi_{i}=\left(u_{i}-k\right)_{+}$in (1.7). The difference appears when we want to estimate the source terms, the boundary conditions and the non-diagonal elements of matrix $\left[a_{i j}\right]$ (see estimates of $I_{2}, I_{3}, I_{4}$ in (3.8)(3.17)). In this case additionally the energy estimate (2.1) is used. Using the test function $\phi_{i}=\left(u_{i}-k\right)_{+}$in $(1.7)$ we are able to obtain $L^{\infty}$-estimate for systems with diagonal main part $\left(a_{i i} \quad 1 \leq i \leq m\right.$ need not to be the same). Similar problem was considered for the Dirichlet boundary condition in $[10]$.

We are not able to prove existence of weak solutions under the assumption (3.1) and in this case only a priori $L^{\infty}$ bound is found under restrictions on growth conditions for nonlinear terms which are listed in Proposition 3.4. Notice that under there assumptions if $\phi_{0}=0$ in (3.1) then it follows the existence of bounded weak solutions.

\section{Existence of solutions}

To show the existence of solutions to (P1) and (P2) we shall use the Galerkin method in much the same way as in [8]. Since some parts of our proof are standard we only give references to this monograph. We shall first show in Theorem 2.3 existence of solutions to $(\mathbf{P 1})$ and then we extend the result in Theorem 2.4 for the case (P2).

It is worth pointing out that the restrictions imposed on the data functions are mainly due to the necessity of showing the strong convergence of gradients of approximating solutions to their weak limits.

We shall use the following auxiliary fact.

Proposition 2.1. Let $W$ be the space defined in (1.6). Then the following embedding is continuous

$$
W \subset C\left([0, T] ; L^{2}(\Omega)\right)
$$

Proof. Our proof requires only a small modification of the proof of $[6$, Theorem 1.17, Ch. 4] therefore we only sketch it. At first, one proves, 
following [6, Lemma 1.12], that the embedding

$$
C^{1}\left([0, T] ; W^{1, p}(\Omega)\right) \cap Y \subset W
$$

is dense. Then, using the integration by parts formula one derives

$$
\begin{aligned}
\|u(t)\|_{2}^{2} & =\int_{0}^{T}\left\{\phi^{\prime}(s)(u(s), u(s))+2 \phi(s)\left(u^{\prime}(s), u(s)\right)\right\} d s \\
& -2 \int_{t}^{T}\left(u^{\prime}(s), u(s)\right) d s,
\end{aligned}
$$

where $u \in C^{1}\left([0, T] ; W^{1, p}(\Omega)\right) \cap Y$ and $\phi$ is an arbitrary function such that $\phi \in C^{1}([0, T]), \phi(0)=0, \phi(T)=1$. Hence, we have

$$
\begin{aligned}
\|u(t)\|_{2}^{2} \leq & \sup _{s \in[0, T]}\left|\phi^{\prime}(s)\right|\|u\|_{X \cap Y}^{2}+2 \sup _{s \in[0, T]}|\phi(s)|\left\|u^{\prime}\right\|_{X^{\prime}+Y^{\prime}} \cdot\|u\|_{X \cap Y} \\
& +2\left\|u^{\prime}\right\|_{X^{\prime}+Y^{\prime}} \cdot\|u\|_{X \cap Y} \leq \text { const }\|u\|_{W}^{2} .
\end{aligned}
$$

It follows that

$$
\|u\|_{C\left([0, T] ; L^{2}(\Omega)\right)} \leq \text { const }\|u\|_{W}
$$

for $u \in C^{1}\left([0, T] ; W^{1, p}(\Omega)\right) \cap Y$ and by density argument also for $u \in W$.

The following a priori estimate is a main tool for proving existence of weak solutions.

Lemma 2.2. Weak solutions of (P1) satisfy the following a priori estimate

$$
\begin{aligned}
\sum_{i=1}^{m}\left\|u_{i}(t)\right\|_{2}^{2} & +\sum_{i=1}^{m} \iint_{\Omega_{t}}\left|\nabla u_{i}(x, t)\right|^{p} d x d t \\
& +\sum_{i=1}^{m} \iint_{\Omega_{t}}\left|u_{i}(x, t)\right|^{p_{0}} d x d t \leq C_{1} .
\end{aligned}
$$

Proof. Multiplying $i$-th equation in (1.1) by $u_{i}$, integrating on $\Omega_{t}$ and then using (H1), (H2), (H3) and (H4) we obtain

$$
\begin{aligned}
& \frac{1}{2} \sum_{i=1}^{m}\left\|u_{i}(\cdot, t)\right\|_{2}^{2}+\underline{\alpha} \sum_{i=1}^{m} \iint_{\Omega_{t}}\left|\nabla u_{i}\right|^{p}+\underline{\beta} \sum_{i=1}^{m} \iint_{\Omega_{t}}\left|u_{i}\right|^{p_{0}} \\
& \leq \frac{1}{2} \sum_{i=1}^{m}\left\|u_{0 i}\right\|_{2}^{2}+\gamma \iint_{S_{t}}\left(1+|u|^{b}\right) u \\
& +\delta \iint_{\Omega_{t}}\left(1+|u|^{\mu}+|\nabla u|^{\nu}\right) u:=I_{0}+I_{1}+I_{2} .
\end{aligned}
$$


We will use the following inequality (see [5, p. 22])

$$
\int_{\partial \Omega}|v|^{q} \leq \varepsilon \int_{\Omega}|\nabla v|^{q}+C \varepsilon^{\frac{-1}{q-1}} \int_{\Omega}|v|^{q}, \quad q>1, v \in W^{1, q}(\Omega)
$$

for $\varepsilon$ sufficiently small, $0<\varepsilon \leq \varepsilon_{0}$, where $\varepsilon_{0}$ depends on the boundary of $\Omega$. Using (H3) and (2.3) and then the Young inequality we obtain

$$
I_{1} \leq \varepsilon \iint_{\Omega_{t}}|\nabla u|^{p}+\varepsilon \iint_{\Omega_{t}}|u|^{p_{0}}+C_{\varepsilon}^{1},
$$

for $b+1 \leq p$ if $p_{0}>p$ or for $b+1<p_{0}$ if $2<p_{0} \leq p$ where $C_{\varepsilon}^{1}=$ $C_{\varepsilon}^{1}(\gamma,|\Omega|, t)$. If $p_{0}=2$ we find similarly

$$
I_{1} \leq \varepsilon \iint_{\Omega_{t}}|\nabla u|^{2}+C_{\varepsilon}^{2}\left(\iint_{\Omega_{t}}|u|^{2}+1\right),
$$

where $C_{\varepsilon}^{2}=C_{\varepsilon}^{2}(\gamma,|\Omega|, t ; \varepsilon)$. Using (1.4) and the Young inequality we obtain

$$
I_{2} \leq \varepsilon \iint_{\Omega_{t}}|u|^{p_{0}}+\varepsilon \iint_{\Omega_{t}}|\nabla u|^{p}+C_{\varepsilon}^{3},
$$

where $C_{\varepsilon}^{3}=C_{\varepsilon}^{3}\left(\delta, \mu, \nu, p_{0},|\Omega|, t ; \varepsilon\right)$. If $p_{0} \neq 2$ choosing $\varepsilon$ sufficiently small in (2.4) and in (2.6) we obtain (2.1). If $p_{0}=2$ we arrive at (2.1) using (2.5) and the Gronwall lemma .

Theorem 2.3. There exists a weak solution to (P1).

Proof. Let $\left\{\psi_{k} ; k=1,2, \ldots\right\}$ be a linearly dense system in the space $W^{1, p}(\Omega) \cap L^{p_{0}}(\Omega)$. Assume also that

$$
\left(\psi_{k}, \psi_{i}\right)=\delta_{k, i} \quad \text { and } \quad \max \left\{\left\|\psi_{k}\right\|_{\infty},\left\|\nabla \psi_{k}\right\|_{\infty}\right\} \leq C_{k}
$$

where $k \geq 1$ and $C_{k}$, are positive constants. We are looking for an approximate solution to (1.1) in the form

$$
u_{i}^{N}(x, t)=\sum_{k=1}^{N} c_{k, i}^{N}(t) \psi_{k}(x), \quad i=1,2, \ldots, m ; k=1,2, \ldots, N,
$$

where $c_{k}^{N}(t) \in \mathbb{R}^{\gg}, k=1,2, \ldots, N$ satisfy the following system of ordinary differential equations

$$
\begin{aligned}
& \frac{d}{d t} c_{k, i}^{N}(t)+\left(\sum_{i=1}^{m} a_{i j}\left(\cdot, t, u^{N}(\cdot, t), \nabla u^{N}(\cdot, t)\right) \nabla u_{j}^{N}(\cdot, t), \nabla \psi_{k}\right) \\
& +\left(R_{i}\left(\cdot, t, u^{N}(\cdot, t)\right) u_{i}^{N}(\cdot, t), \psi_{k}\right)=\left(f_{i}\left(\cdot, t, u^{N}(\cdot, t), \nabla u^{N}(\cdot, t), \psi_{k}\right)\right. \\
& +\int_{S} g_{i}\left(\cdot, t, u^{N}(\cdot, t) \psi_{k} d S, \quad k=1,2, \ldots, N, \quad i=1,2, \ldots, m\right.
\end{aligned}
$$


with initial conditions

$$
c_{k, i}^{N}(0)=\left(u_{0 i}, \psi_{k}\right), \quad k=1,2, \ldots, N, \quad i=1,2, \ldots, m,
$$

where $u_{i}^{N}$ is defined in (2.7) and $\nabla u_{i}^{N}(x, t)=\sum_{k=1}^{N} c_{k, i}^{N}(t) \nabla \psi_{k}(x)$.

By the hypotheses (H0)-(H4) and Lemma 2.2 it follows that system (2.8) has at least one solution in the sense of Carathéodory determined on $[0, T]$ such that

$$
\begin{aligned}
& \operatorname{ess} \sup _{t \in[0, T]} \sum_{i=1}^{m}\left\|u_{i}^{N}(t)\right\|_{2}^{2}+\underline{\alpha} \sum_{i=1}^{m} \iint_{\Omega_{T}}\left|\nabla u_{i}^{N}(x, t)\right|^{p} d x d t \\
& +\underline{\beta} \sum_{i=1}^{m} \iint_{\Omega_{T}}\left|u_{i}^{N}(x, t)\right|^{p_{0}} d x d t \leq C_{1},
\end{aligned}
$$

where $C_{1}$ is a constant independent of $N$. Notice that if $2<p_{0}<p$ then by $(1.7) u^{N}$ is also uniformly bounded in $L^{p}\left(\Omega_{T}\right)$. Hence, by the weak compactness, there exists a subsequence still denoted by $\left(u^{N}\right)_{N \geq 1}$ and $u \in L^{p}\left(0, T ; W^{1, p}(\Omega)\right) \cap L^{p_{0}}\left(\Omega_{T}\right)$ such that

$$
\begin{aligned}
& u^{N} \rightarrow u \quad \text { weakly in } L^{p_{0}}\left(\Omega_{T}\right) \quad \text { as } \quad N \rightarrow \infty \\
& \nabla u^{N} \rightarrow \nabla u \quad \text { weakly in } L^{p}\left(\Omega_{T}\right) \text { as } \quad N \rightarrow \infty .
\end{aligned}
$$

We shall show that for a subsequence

$$
u^{N}(t) \rightarrow u(t) \quad \text { weakly in } \quad L^{2}(\Omega) \quad \text { as } \quad N \rightarrow \infty
$$

uniformly with respect to $t \in[0, T]$. Applying for fixed $k$ the Hölder inequality we obtain

$$
\begin{aligned}
& \left|c_{k, i}^{N}(t+\Delta t)-c_{k, i}^{N}(t)\right|=\left|\left(u_{i}^{N}(x, t+\Delta t)-u_{i}^{N}(x, t), \psi_{k}(x)\right)\right|(2.14) \\
\leq & \int_{t}^{t+\Delta t} \int_{\Omega}\left|\sum_{j=1}^{m} a_{i j}\left(x, s, u^{N}, \nabla u^{N}\right) \nabla u_{j} \nabla \psi_{k}\right| d x d s \\
+ & \sum_{i=1}^{m}\left(\int_{t}^{t+\Delta t} \int_{\Omega}\left|R_{i}\left(x, s, u^{N}\right) u_{i}^{N} \psi_{k}\right| d x d s\right. \\
+ & \int_{t}^{t+\Delta t} \int_{\Omega}\left|f_{i}\left(x, s, u^{N}, \nabla u^{N}\right) \psi_{k}\right| d x d s+\int_{t}^{t+\Delta t} \int_{\partial \Omega}\left|g_{i}(x, s, u) \psi_{k}\right| d S d s \\
\leq & C_{k}^{\prime}\left\{\Delta t|\Omega|+\left\|u^{N}\right\|_{X}(\Delta t|\Omega|)^{1 /(p-1)}+\left\|u^{N}\right\|_{Y}(\Delta t|\Omega|)^{1 /\left(p_{0}-1\right)}\right\} \\
\leq & \varepsilon_{k}(\Delta t)
\end{aligned}
$$

where $\varepsilon_{k}$ is independent of $N$ thanks to $(2.10)$ and $\varepsilon_{k}(h) \rightarrow 0$ as,$h \rightarrow 0$. Now using the same arguments as in $[8$, Theorem $4.1, \mathrm{Ch}$. III] we arrive at (2.13). 
Applying [8, Lemma 6.1, Ch. V] to $u_{i}^{N}-u_{i}^{M} \in L^{p}\left(0, T ; W^{1, p}(\Omega)\right)$ we have for arbitrary $\varepsilon>0$

$$
\begin{aligned}
& \int_{0}^{T}\left\|u_{i}^{N}(t)-u_{i}^{M}(t)\right\|_{2}^{2} d t \leq \int_{0}^{T} \sum_{k=1}^{N_{\varepsilon}}\left(u_{i}^{N}(t)-u_{i}^{M}(t), \psi_{k}\right)^{2} d t \\
& +\varepsilon\left\|u_{i}^{N}-u_{i}^{M}\right\|_{L^{p}\left(0, T ; W^{1, p}(\Omega)\right)}:=I_{1}+I_{2},
\end{aligned}
$$

for $i=1,2, \ldots, m$, where $p>2 n /(n+2)$ for $n \geq 2$ and $p \geq 1$ for $n=1$. In view of (2.1) and (2.15) for $N$ and $M$ sufficiently large $I_{1}<\varepsilon$ and $I_{2}<\varepsilon 2 C_{1}$. Hence, for a subsequence

$$
u_{i}^{N} \rightarrow u_{i} \quad \text { in } L^{2}\left(\Omega_{T}\right), \quad i=1,2, \ldots, m
$$

and

$$
u_{i}^{N} \rightarrow u \quad \text { a.e. in } \Omega_{T} .
$$

By [5, Lemma 3, p. 10] one obtains

$$
u_{i} \rightarrow u \text { strongly in } L^{s}\left(S_{T}\right),
$$

where $0<s<p+2(p-1) / n$. Let us denote $V_{k}=\operatorname{span}\left\{\psi_{1}, \ldots, \psi_{k}\right\}$ and

$$
X_{k}=L^{p}\left(0, T ; V_{k}\right), \quad Y_{k}=L^{p_{0}}\left(0, T ; V_{k}\right) .
$$

From (2.8) it follows that for $i=1,2, \ldots, m$ and $N \geq k$

$$
\begin{aligned}
\int_{0}^{T}\left(\frac{d u_{i}^{N}}{d t}, \varphi_{i}\right) d t+\int_{0}^{T} & \left(\sum_{i=1}^{m} a_{i j}\left(u^{N}, \nabla u^{N}\right) \nabla u_{j}^{N}, \nabla \varphi_{i}\right) d t \\
+\int_{0}^{T}\left(R_{i}\left(u^{N}\right) u_{i}^{N}, \varphi_{i}\right) d t & =\int_{0}^{T}\left(f_{i}\left(u^{N}, \nabla u^{N}\right), \varphi_{i}\right) d t \\
+ & \iint_{S_{T}} g_{i}\left(u^{N}\right) \varphi_{i} d S d t
\end{aligned}
$$

for all $\varphi_{i} \in X_{k} \cap Y_{k}$. Notice that

$$
R_{i}\left(u^{N}\right) u_{i}^{N} \rightarrow R_{i}(u) u_{i} \quad \text { weakly in } \quad L^{p_{0}^{*}}\left(\Omega_{T}\right), \quad \frac{1}{p_{0}}+\frac{1}{p_{0}^{*}}=1
$$

which follows from (2.17), (2.10) and (H2.i). Similar argument yields by (2.18)

$$
g_{i}\left(u^{N}\right) \rightarrow g_{i}(u) \quad \text { weakly in } \quad L^{p^{*}}\left(S_{T}\right), \quad \frac{1}{p}+\frac{1}{p^{*}}=1
$$

Letting $N \rightarrow \infty$ we obtain

$$
\int_{0}^{T}\left(u_{i}^{\prime}, \varphi_{i}\right) d t+\int_{0}^{T}\left(\sum_{i=1}^{m} \xi_{i j}, \nabla \varphi_{i}\right) d t+\int_{0}^{T}\left(R(u) u_{i}, \varphi_{i}\right) d t
$$




$$
=\int_{0}^{T}\left(\zeta_{i}, \varphi_{i}\right) d t+\iint_{S_{T}} g_{i}(u) \varphi_{i} d S d t
$$

for all $\varphi_{i} \in X_{k} \cap Y_{k}$, where $\xi_{i j}$ and $\zeta_{i}$ are weak limits of $a_{i j}\left(x, t, u^{N}, \nabla u^{N}\right)$ and $f_{i}\left(x, t, u^{N}, \nabla u^{N}\right)$ respectively, for $i=1,2, \ldots, m, j=1,2, \ldots, n$. By $\left[6\right.$, Lemma 1.5 , Ch. VI] the set $\bigcup_{N=1}^{\infty} C^{1}\left(0, T ; V_{N}\right)$ is dense in $C^{1}([0, T]$; $\left.\left.W^{1, p}(\Omega)\right) \cap Y\right)$. Following the proof of $[6$, Lemma 1.12 , Ch. IV] one also shows that the latter space is dense in both $X \cap Y$ and $W$. Consequently (2.20) is also true for all $\varphi_{i} \in X \cap Y$.

To complete the proof we shall show that $\nabla u_{i}^{N} \rightarrow \nabla u_{i}$ strongly in $L^{p}\left(\Omega_{T}\right)$. Let $\left\{w^{N}\right\}_{N=1}^{\infty}$ be an arbitrary sequence such that

$$
\begin{aligned}
& w^{N} \in\left(C^{1}\left([0, T] ; V_{N}\right)\right)^{m} \quad \text { and } \\
& w^{N} \rightarrow u \text { in } W^{m} .
\end{aligned}
$$

Setting $\varphi_{i}=\left.\left(u_{i}^{N}-w_{i}^{N}\right)\right|_{[0, t]}:=v_{i}^{N}$ in $(2.20)$ and using $(\mathbf{H 1} . \mathbf{i})$ we obtain

$$
\begin{aligned}
& \frac{1}{2} \sum_{i=1}^{m}\left\|v_{i}^{N}(t)\right\|_{2}^{2}+\underline{\alpha} \sum_{i=1}^{m} \iint_{\Omega_{t}}\left|\nabla v_{i}^{N}\right|^{p}+\underline{\beta} \sum_{i=1}^{m} \iint_{\Omega_{t}}\left|v_{i}\right|^{p_{0}} \\
& \quad \leq \frac{1}{2} \sum_{i=1}^{m}\left\|v_{i}^{N}(0)\right\|_{2}^{2}-\sum_{i=1}^{m} \int_{0}^{t}\left(\hat{w}_{i}^{N}(s), v_{i}^{N}(s)\right) d s \\
& \quad-\sum_{i, j=1}^{m} \iint_{\Omega_{t}} a_{i j}\left(u^{N}, \nabla w^{N}\right) \nabla w_{j}^{N} \nabla v_{i}^{N} \\
& \quad-\sum_{i=1}^{m} \iint_{\Omega_{t}} R_{i}\left(w^{N}\right) w_{i}^{N} v_{i}^{N}+\sum_{i=1}^{m} \iint_{\Omega_{t}} f_{i}\left(u^{N}, \nabla u^{N}\right) v_{i}^{N} \\
& \quad+\sum_{i=1}^{m} \iint_{S_{t}} g_{i}\left(u^{N}\right) v_{i}^{N} .
\end{aligned}
$$

By (2.21) and Lemma 2.1

$$
w_{i}^{N}(0) \rightarrow u_{i}(0) \quad \text { in } L^{2}(\Omega) \quad \text { as } N \rightarrow \infty .
$$

Hence, using (2.9)

$$
\sum_{i=1}^{m}\left\|v_{i}^{N}(0)\right\|_{2}^{2} \rightarrow 0 \quad \text { as } N \rightarrow \infty .
$$

Since $v_{i}^{N} \rightarrow 0$ weakly in $X \cap Y$ and (2.21)

$$
\sum_{i=1}^{m} \int_{0}^{T}\left(\dot{w}_{i}^{N}(s), v_{i}^{N}(s)\right) d s \rightarrow 0 \quad \text { as } N \rightarrow \infty .
$$


Proceeding as in [10] we have

$$
\begin{aligned}
& \left|\iint_{\Omega_{t}} \sum_{i, j=1}^{m} a_{i j}\left(u^{N}, \nabla w^{N}\right) \nabla w_{j}^{N} \nabla v_{i}^{N} d x d t\right| \\
& \leq\left|\iint_{\Omega_{t}} \sum_{i, j=1}^{m}\left\{a_{i j}\left(u^{N}, \nabla w^{N}\right) \nabla w_{j}^{N}-a_{i j}(u, \nabla u) \nabla u_{j}\right\} \nabla v_{i}^{N}\right| \\
& +\left|\iint_{\Omega_{t}} \sum_{i, j=1}^{m} a_{i j}(u, \nabla u) \nabla u_{j} \nabla v_{i}^{N}\right| \leq \eta_{1} \iint_{\Omega_{t}}\left|\nabla v_{i}^{N}\right|^{p} \\
& +C_{\eta_{1}} \iint_{\Omega_{t}}\left(\sum_{i, j=1}^{m} a_{i j}\left(u^{N}, \nabla w^{N}\right) \nabla w_{j}^{N}-a_{i j}(u, \nabla u) \nabla u_{j}\right)^{p /(p-1)} \\
& +\iint_{\Omega_{t}} \sum_{i, j=1}^{m} a_{i j}(u, \nabla u) \nabla u_{j} \nabla v_{i}^{N},
\end{aligned}
$$

where $\eta_{1}>0$.

The Nemytskii operator $A(\phi, \xi):=\sum_{i, j=1}^{m} a_{i j}(\phi, \xi) \xi_{j}$ maps $\left(L^{p}\left(\Omega_{T}\right)\right)^{m} \times\left(L^{p}\left(\Omega_{T}\right)\right)^{m n}$ into $L^{p /(p-1)}\left(\Omega_{T}\right)$ and it is continuous (see e.g. [6]). Therefore,

$$
\iint_{\Omega_{t}}\left(\sum_{i, j=1}^{m}\left(a_{i j}\left(u^{N}, \nabla w^{N}\right) \nabla w_{j}^{N}-a_{i j}(u, \nabla u) \nabla u_{j}\right)\right)^{p /(p-1)} \rightarrow 0 \text { as } N \rightarrow \infty .
$$

Since $a_{i j}(u, \nabla u) \nabla u_{j} \in L^{p /(p-1)}\left(\Omega_{T}\right)$ and $v_{i}^{N} \rightarrow 0$ weakly in $X \cap Y$ we have also

$$
\iint_{\Omega_{t}} \sum_{i, j=1}^{m} a_{i j}(u, \nabla u) \nabla u_{j} \nabla v_{i}^{N} \rightarrow 0 \quad \text { as } N \rightarrow \infty .
$$

We proceed in much the same way with forth term on the r.h.s. of $(2.22)$. Thus,

$$
\begin{aligned}
& \left|\sum_{i=1}^{m} \iint_{\Omega_{t}} R_{i}\left(w^{N}\right) w_{i}^{N} v_{i}^{N}\right| \leq \eta_{2} \iint_{\Omega_{t}} \sum_{i=1}^{m}\left|v_{i}^{N}\right|^{p_{0}} \\
& +C_{\eta_{2}} \iint_{\Omega_{t}}\left(\sum_{i=1}^{m} R_{i}\left(w^{N}\right) w_{i}^{N}-R_{i}(u) u_{i}\right)^{p_{0} /\left(p_{0}-1\right)}+\iint_{\Omega_{t}} \sum_{i=1}^{m} R_{i}(u) u_{i} v_{i}^{N},
\end{aligned}
$$


where $\eta_{2}>0$. Similar arguments as in the previous case yield

$$
\iint_{\Omega_{t}}\left(\sum_{i=1}^{m} R_{i}\left(w^{N}\right) w_{i}^{N}-R_{i}(u) u_{i}\right)^{p_{0} /\left(p_{0}-1\right)} \rightarrow 0 \quad \text { as } \quad N \rightarrow \infty
$$

and

$$
\iint_{\Omega_{t}} \sum_{i=1}^{m} R_{i}(u) u_{i} v_{i}^{N} \rightarrow 0 \quad \text { as } \quad N \rightarrow \infty .
$$

Notice that by (2.10) and (2.17)

$$
v_{i}^{N} \rightarrow 0 \quad \text { in } \quad L^{r}\left(\Omega_{T}\right) \quad \text { for } \quad r<p_{0}
$$

and by (2.18)

$$
v_{i}^{N} \rightarrow 0 \quad \text { in } L^{p}\left(S_{T}\right) .
$$

Taking $r<p_{0}$ such that $1 / r+\nu / r \leq 1, \mu+1 \leq r$ and using (2.10) and the Hölder inequality yields

$$
\sum_{i=1}^{m} \iint_{\Omega_{t}} f_{i}\left(u^{N}, \nabla u^{N}\right) v_{i}^{N} d x d t \leq C_{1} \sum_{i=1}^{m}\left\|v_{i}^{N}\right\|_{L^{r}\left(\Omega_{T}\right)}
$$

and

$$
\sum_{i=1}^{m} \iint_{S_{T}} g_{i}\left(u^{N}\right) v_{i}^{N} d S d t \leq C_{2} \sum_{i=1}^{m}\left\|v_{i}^{N}\right\|_{L^{p}\left(S_{T}\right)} .
$$

where $C_{1}$ and $C_{2}$ are positive constants. Hence,

$$
\sum_{i=1}^{m} \iint_{\Omega_{t}} f_{i}\left(u^{N}, \nabla u^{N}\right) v_{i}^{N} d x d t \rightarrow 0 \quad \text { as } \quad N \rightarrow \infty
$$

and

$$
\sum_{i=1}^{m} \iint_{S_{T}} g_{i}\left(u^{N}\right) v_{i}^{N} d S d t \rightarrow 0 \quad \text { as } \quad N \rightarrow \infty .
$$

Thus, comming back to $(2.22)$ we conclude that $\nabla u^{N} \rightarrow \nabla u$ in $L^{p}\left(\Omega_{T}\right)$ and consequently

$$
\xi_{i j}=a_{i j}(x, t, u, \nabla u) \nabla u_{j} \quad \text { and } \quad \zeta_{i}=f_{i}(x, t, u, \nabla u)
$$

for $i=1,2, \ldots, m, j=1,2, \ldots, n$. It follows from Lemma 2.1 that $u_{i} \in$ $C\left([0, T] ; L^{2}(\Omega)\right)$. This completes the proof.

Theorem 2.4. There exists a weak solution to (P2). 
Proof. In order to show the apriori estimate we proceed step by step starting from the last equation. Proceeding analogously to the proof of Lemma 2.1 using $(\mathbf{H 2} . \mathbf{i})^{\prime},(\mathbf{H 2} . \mathbf{i i})^{\prime},(\mathbf{H} 3),(\mathbf{H} 4)$ we obtain for the $m$-th equation

$$
\begin{aligned}
& \frac{1}{2}\left\|u_{m}(t)\right\|_{2}^{2}+\alpha_{0} \iint_{\Omega_{t}}\left(\left|\nabla u_{m}\right|^{p}+\left|u_{m}\right|^{p_{0}}\right) \\
& \leq \varepsilon \sum_{i=1}^{m} \iint_{\Omega_{t}}\left(\left|\nabla u_{i}\right|^{p}+\left|u_{i}\right|^{p_{0}}\right) \\
& +C_{\varepsilon}^{(2)}\left(1+\frac{1}{2} \sum_{i=1}^{m}\left(\left\|u_{i}(0)\right\|_{2}^{2}+\iint_{\Omega_{t}}\left|u_{i}\right|^{2}\right)\right):=\varepsilon Q+C_{\varepsilon}^{(2)} R,
\end{aligned}
$$

where $\alpha_{0}=\min (\underline{\alpha}, \underline{\beta}), \quad \varepsilon>0$ and $C_{\varepsilon}^{(2)}=C_{\varepsilon}^{(2)}\left(\delta, \gamma, \mu, \nu,, p_{0}\right)$. For $(m-1)$-th equation one obtains

$$
\begin{aligned}
& \frac{1}{2}\left\|u_{m-1}(t)\right\|_{2}^{2}+\underline{\alpha} \iint_{\Omega_{t}}\left|\nabla u_{m-1}\right|^{p} \\
& +\iint_{\Omega_{t}}\left(R_{m-1}^{I}\left(u_{m-1}\right) u_{m-1}^{2}+R_{m-1}^{I I}\left(u_{m}\right) u_{m-1}^{2}\right) \\
& \leq \iint_{\Omega_{t}}\left|a_{m-1, m}\left(u_{m}, \nabla u_{m}\right) \nabla u_{m} \nabla u_{m-1}\right|+\left|f_{m-1}(u, \nabla u) u_{m-1}\right| \\
& +\iint_{S_{T}}\left|g(u) u_{m-1}\right| d S d t .
\end{aligned}
$$

Using (2.23) and $(\text { H1.ii })^{\prime},(\text { H2.i })^{\prime},(\text { H2.ii })^{\prime},($ H3), $($ H4) we arrive at

$$
\begin{aligned}
& \frac{1}{2}\left\|u_{m-1}(t)\right\|_{2}^{2}+\alpha_{0} \iint_{\Omega_{t}}\left(\left|\nabla u_{m-1}\right|^{p}+\left|u_{m-1}\right|^{p_{0}}\right) \\
& \leq \varepsilon Q+C_{\varepsilon}^{(1)} \iint_{\Omega_{t}}\left(\left|\nabla u_{m}\right|^{p}+\left|u_{m}\right|^{p_{0}}\right)+C_{\varepsilon}^{(2)} R \\
& \leq\left(\varepsilon Q+C_{\varepsilon}^{(2)} R\right) d,
\end{aligned}
$$

where $C_{\varepsilon}^{(1)}=C_{\varepsilon}^{(1)}\left(\alpha_{0}, \bar{\beta}\right)$ is a constant and $d=1+C_{\varepsilon}^{(1)} / \alpha_{0}$. Finally we find by induction

$$
\begin{aligned}
& \sum_{i=1}^{m}\left\{\frac{1}{2}\left\|u_{i}(t)\right\|_{2}^{2}+\alpha_{0} \iint_{\Omega_{t}}\left(\left|\nabla u_{i}\right|^{p}+\left|u_{i}\right|^{p_{0}}\right)\right\} \\
& \leq\left(\varepsilon Q+C_{\varepsilon}^{(2)} R\right)(d+1)^{m-1} .
\end{aligned}
$$

Taking $\varepsilon<\alpha_{0} /(1+d)^{m-1}$ and applying the Gronwall lemma we obtain desired estimate.

Except of the last part of the proof related to the strong convergence of gradients other steps of the proof are the same as that of Theorem 2.3. At 
first one shows that for $i=m$

$$
\nabla u_{i}^{N} \rightarrow \nabla u_{i} \text { in } L^{p}\left(\Omega_{T}\right) \text { and } \quad u_{i}^{N} \rightarrow u_{i} \text { in } L^{p_{0}}\left(\Omega_{T}\right) \text { as } N \rightarrow \infty
$$

by repeating the reasoning used in the proof of Theorem 2.3. Using this fact in $(m-1)$-th equation one proves convergence in $(2.26)$ for $i=m-1$ and so on from $i=m-1$ to $i=1$ which completes the proof.

Remark 2. One can assume in $(\mathbf{H 0})^{\prime}$ that $a_{i j}$ depends on all variables $u_{1}, \ldots u_{m}$ for $m \geq j>i \geq 1$. In this case, however, one has to exclude the case $r=p_{0}(1-1 / p)$.

\section{A priori $L^{\infty}$ bounds for the weak solutions}

In this section we make the following assumptions:

$$
a_{i i}(\cdot, u, q) \geq \underline{\alpha}\left|q_{i}\right|^{p-2}-\phi_{0}(x, t), \quad i=1, \cdots, m,
$$

a.e. in $\Omega_{T}$ for $u \in \mathbb{R}^{\gg}, q \in \mathbb{R}^{\gg \ltimes}, q=\left(q_{1}, \ldots, q_{m}\right)$ where $\phi_{0} \in L^{\sigma}\left(\Omega_{T}\right), \quad \sigma>$ $p /(p-2)$ and $\phi_{0}(x, t) \geq 0$ a.e. in $\Omega_{T}$ and

$$
\left|a_{i j}(\cdot, u, q)\right| \leq \bar{\alpha}\left(1+|u|^{r_{1}}+|q|^{r_{2}}\right) \quad \text { for } i \neq j, \quad i, j=1, \ldots, m,
$$

with nonnegative constants $r_{1}, r_{2}$ which will be specified later.

Let $A_{k, i}^{+}(t)=\left\{x \in \bar{\Omega}: u_{i}(x, t)>k\right\}$ a.e. in $[0, T], i=1, \ldots, m$. It is easy to check that under the assumptions (3.1), (3.2) the energy estimate (2.1) still holds. To show $L^{\infty}$-estimate we need

Lemma 3.1. Let $u_{i} \in W, i=1, \ldots, m$, be a solution to the problem either (P1) or (P2) supplemented by the assumptions (3.1) and (3.2). Assume that

$$
\begin{aligned}
& b<p-1 \\
& \mu<p^{*}, \quad \nu<p \\
& r_{1}<p^{*}\left(1-\frac{2}{p}\right), \quad r_{2}<p-2,
\end{aligned}
$$

where $p^{*}=\max \left\{q, p_{0}\right\}$ and $q=p(n+2) / n$.

Let $\bar{k}$ be a positive number such that

$$
\bar{k} \geq\left\|u_{0}\right\|_{\infty} .
$$

Then the following estimate holds

$$
\sum_{i=1}^{m}\left[\operatorname{ess} \sup _{t \in[0, T]} \int_{\Omega}\left(u_{i}(x, t)-\bar{k}\right)_{+}^{2} d x+2 \underline{\alpha} \iint_{\Omega_{T}}\left|\nabla\left(u_{i}-\bar{k}\right)_{+}\right|^{p} d x d t\right.
$$




$$
\begin{aligned}
& \left.+2 \underline{\beta} \iint_{\Omega_{T}}\left(u_{i}-\bar{k}\right)_{+}^{p_{0}} d x d t\right] \leq C_{0} \sum_{i=1}^{m}\left[\iint_{\Omega_{T}}\left(u_{i}-\bar{k}\right)_{+}^{p} d x d t\right. \\
& \sum_{j=1}^{2}\left(\iint_{\Omega_{T}}\left(u_{i}-\bar{k}\right)_{+}^{\sigma_{j}} d x d t\right)^{1 / \sigma_{j}}+\iint_{\Omega_{T}}\left(u_{i}-\bar{k}\right)_{+} d x d t \\
& \left.+\sum_{j=1}^{4}\left(\int_{0}^{T}\left|A_{\bar{k}, i}(t)\right| d t\right)^{1-\gamma_{j}}\right],
\end{aligned}
$$

where $\sigma_{1}=p^{*} /\left(p^{*}-\mu\right), \sigma_{2}=p /(p-\nu), \gamma_{1}=\left(r_{1} p_{1}+p^{*}\right) /\left[p^{*}(p-1)\right], \gamma_{2}=$ $\left(r_{2}+1\right) /(p-1), \gamma_{3}=(b+1) / p, \gamma_{4}=p /[\sigma(p-2)]$ and $C_{0}$ is a positive constant depending on the data.

Proof. Using (3.1), (3.2) and testing (1.7) with $\varphi_{i}=\left(u_{i}-\bar{k}\right)_{+}, i=1,2$, $\ldots, m$, we obtain

$$
\begin{aligned}
& \sum_{i=1}^{m}\left[\sup _{t \in[0, T]} \int_{\Omega}\left(u_{i}-\bar{k}\right)_{+}^{2} d x+2 \underline{\alpha} \iint_{\Omega_{T}}\left|\nabla\left(u_{i}-\bar{k}\right)_{+}\right|^{p} d x d t\right. \\
& \left.+\int_{\Omega_{T}} R_{i}(u) u_{i}\left(u_{i}-\bar{k}\right)_{+} d x d t\right] \leq \sum_{i=1}^{m}\left[\iint_{\Omega_{T}} \phi_{0}\left|\nabla\left(u_{i}-\bar{k}\right)_{+}\right|^{2} d x d t\right. \\
& \left.+\int_{S_{T}} g_{i}(x, t, u)\left(u_{i}-\bar{k}\right)_{+} d S d t+\iint_{\Omega_{T}} f_{i}(x, t, u, \nabla u)\left(u_{i}-\bar{k}\right)_{+} d x d t\right] \\
& +\sum_{i, j=1}^{m} \iint_{\Omega_{T}}\left(1-\delta_{i j}\right) a_{i j}(x, t, u, \nabla u) \nabla u_{j} \nabla\left(u_{i}-\bar{k}\right)_{+} d x d t \equiv \sum_{i=1}^{4} I_{i} .
\end{aligned}
$$

Using (H2.ii) for the last term on the 1.h.s. we obtain

$$
\begin{aligned}
& \sum_{i=1}^{m} \iint_{\Omega_{T}} R_{i}(u) u_{i}\left(u_{i}-\bar{k}\right)_{+} d x d t \\
& \geq \underline{\beta} \sum_{i=1}^{m} \int_{0}^{T} \int_{A_{\bar{k}, i}^{+}(t)}|u|^{p_{0}-2} u_{i}\left(u_{i}-\bar{k}\right)_{+} d x d t \\
& \geq \underline{\beta} \sum_{i=1}^{m} \iint_{\Omega_{T}}\left(u_{i}-\bar{k}\right)_{+}^{p_{0}} d x d t .
\end{aligned}
$$

Hölder's inquality applied to the first term on the r.h.s. of (3.7) yields

$$
I_{1} \leq \varepsilon \int_{\Omega_{T}}\left|\nabla\left(u_{i}-k\right)_{+}\right|^{p} d x d t+c(\varepsilon) \int_{\Omega_{T}}\left|\phi_{0}\right|^{p /(p-2)} \chi_{\left\{u_{i}>k\right\}} d x d t
$$


where the second integral is estimated by

$$
\left\|\phi_{0}\right\|_{L_{\sigma}\left(\Omega^{T}\right)}\left(\int_{0}^{T}\left|A_{k, i}^{+}(t)\right| d t\right)^{1-p /[\sigma(p-2)]}
$$

Using (1.8), (1.9) and (2.1) we find

$$
\begin{aligned}
& I_{2} \leq \gamma \sum_{i=1}^{m}\left(\int_{S_{T}}\left(1+|u|^{b}\right)^{p / b} d S d t\right)^{b / p}\left(\int_{S_{T}}\left(u_{i}-\bar{k}\right)_{+}^{p} d S d t\right)^{1 / p} \\
& \times\left(\int_{0}^{T}\left|A_{\bar{k}, i}^{+}(t)\right| d t\right)^{1-(b+1) / p} \leq K\left(\int_{0}^{T}\left|A_{\bar{k}, i}^{+}(t)\right| d t\right)^{1-(b+1) / p} .
\end{aligned}
$$

where $K$ denotes a positive constant depending on the data. Using (H4) we obtain

$$
I_{3} \leq \delta \sum_{i=1}^{m} \int_{\Omega_{T}}\left(1+|u|^{\mu}+|\nabla u|^{\nu}\right)\left(u_{i}-\bar{k}\right)_{+} d x d t .
$$

Hölder's inequality and (3.4) implies

$$
\begin{aligned}
& \int_{\Omega_{T}}|u|^{\mu}\left(u_{i}-\bar{k}\right)_{+} d x d t \\
& \leq\left(\int_{\Omega_{T}}|u|^{p^{*}} d x d t\right)^{\mu / p^{*}}\left(\int_{\Omega_{T}}\left(u_{i}-\bar{k}\right)^{p^{*} /\left(p^{*}-\mu\right)} d x d t\right)^{\left(p^{*}-\mu\right) / p^{*}} \\
& \leq K\left(\int_{\Omega_{T}}\left(u_{i}-\bar{k}\right)_{+}^{\sigma_{1}}\right)^{1 / \sigma_{1}}
\end{aligned}
$$

where $\sigma_{1}=p^{*} /\left(p^{*}-\mu\right)$, and

$$
\begin{aligned}
& \int_{\Omega_{T}}|\nabla u|^{\nu}\left(u_{i}-\bar{k}\right)_{+} d x d t \\
& \leq\left(\int_{\Omega_{T}}|\nabla u|^{p} d x d t\right)^{\nu / p}\left(\int_{\Omega_{T}}\left(u_{i}-\bar{k}\right)_{+}^{\sigma_{2}} d x d t\right)^{1 / \sigma_{2}} \\
& \leq K\left(\int_{\Omega_{T}}\left(u_{i}-\bar{k}\right)_{+}^{\sigma_{2}}\right)^{1 / \sigma_{2}}
\end{aligned}
$$

where $\sigma_{2}=p /(p-\nu)$. From (3.2) we have

$$
I_{4} \leq \bar{\alpha} \sum_{i, j} \iint_{\Omega_{T}}\left(1+|u|^{r_{1}}+|\nabla u|^{r_{2}}\right) \nabla u_{j} \nabla\left(u_{i}-\bar{k}\right)_{+} d x d t .
$$

By the Young inequality and (3.5) we find

$$
\iint_{\Omega_{T}} \nabla u_{j} \nabla\left(u_{i}-\bar{k}\right)_{+} d x d t \leq
$$




$$
\begin{aligned}
& \leq C_{\varepsilon}\left(\iint_{\Omega_{T}}|\nabla u|^{p}\right)^{1 /(p-1)}\left(\int_{0}^{T}\left|A_{\bar{k}, i}^{+}(t)\right| d t\right)^{1-1 /(p-1)} \\
& +\varepsilon \iint_{\Omega_{T}}\left|\nabla\left(u_{i}-k\right)_{+}\right|^{p} .
\end{aligned}
$$

for $\varepsilon>0$ and

$$
\begin{aligned}
& \iint_{\Omega_{T}}|u|^{r_{1}} \nabla u_{j} \nabla\left(u_{i}-\bar{k}\right)_{+} d x d t \\
& \leq C_{\varepsilon}\left(\iint_{\Omega_{T}}|u|^{p^{*}} d x d t\right)^{r_{1} p^{\prime} / p^{*}}\left(\iint_{\Omega_{T}}|\nabla u|^{p} d x d t\right)^{1 /(p-1)}+\varepsilon \iint_{\Omega_{T}}\left|\nabla\left(u_{i}-\bar{k}\right)_{+}\right|^{p}, \\
& \times\left(\int_{0}^{T}\left|A_{\bar{k}, i}^{+}(t)\right| d t\right)^{1-\left(r_{1} p+p^{*}\right) /\left[p^{*}(p-1)\right]}
\end{aligned}
$$

where $1 / p+1 / p^{\prime}=1$, and similarly from (3.5) it follows that

$$
\begin{aligned}
& \iint_{\Omega_{T}}|\nabla u|^{r_{2}} \nabla u_{j} \nabla\left(u_{i}-\bar{k}\right)_{+} d x d t \leq \varepsilon \iint_{\Omega_{T}}\left|\nabla\left(u_{i}-\bar{k}\right)_{+}\right|^{p} \\
& +c_{\varepsilon}\left(\iint_{\Omega_{T}}|\nabla u|^{p} d x d t\right)^{\left(r_{2}+1\right) /(p-1)}\left(\int_{0}^{T}\left|A_{\bar{k}, i}^{+}(t)\right| d t\right)^{1-\left(r_{2}+1\right) /(p-1)} .
\end{aligned}
$$

Finally combining (3.14)-(3.16) we obtain

$$
\begin{gathered}
I_{4} \leq C_{\varepsilon}^{(4)}\left(\sum_{i=1}^{m}\left(\int_{0}^{T}\left|A_{\bar{k}, i}^{+}(t)\right| d t\right)^{1-1 /(p-1)}+\left(\int_{0}^{T}\left|A_{\bar{k}, i}^{+}(t)\right| d t\right)^{1-\left(r_{1} p+p^{*}\right) /\left[p^{*}(p-1)\right]}\right. \\
\left.+\left(\int_{0}^{T}\left|A_{\bar{k}, i}^{+}(t)\right| d t\right)^{1-\left(r_{2}+1\right)(p-1)}\right)+3 \varepsilon \sum_{i=1}^{m} \iint_{\Omega_{T}}\left|\nabla\left(u_{i}-k\right)_{+}\right|^{p} d x d t
\end{gathered}
$$

with a positive constant $C^{(4)}$ depending only on the data. Setting $\varepsilon=\underline{\alpha} / 6$ we arrive at (3.8). This completes the lemma.

Let us define $k_{s}=\left\|u_{0}\right\|_{L^{\infty}\left(\Omega_{T}\right)}+k-k / 2^{s}, k>0, s=0,1, \ldots, \tilde{k}_{s}=$ $\left(k_{s}+k_{s+1}\right) / 2=\left\|u_{0}\right\|_{L^{\infty}\left(\Omega_{T}\right)}+k-3 k / 2^{s+1}$ and

$$
Y_{s}=\sum_{i=1}^{m} \int_{\Omega_{T}}\left(u_{i}-k_{s}\right)_{+}^{\delta} d x d t
$$

where $\delta<q$.

Lemma 3.2. The following recursive inequality holds

$$
Y_{s+1} \leq c_{*} \frac{2^{s b^{*}}}{k^{b_{*}}} Y_{s}^{1+\alpha},
$$


where $b^{*}=\left[1+a^{*}(n+p) / n\right] \delta / 2+\delta(1-\delta / 2), b_{*}=\left[1+a_{*}(n+p) / n\right] \delta / 2+$ $\delta(1-\delta / 2), \alpha=\left(1-\gamma_{*}\right)[(n+p) / n] \delta / q-\delta / q$, where $a^{*}, a_{*}$ and $\gamma_{*}$ are defined by (3.22) and $c_{*}$ depends on the data from the energy estimate (2.1), and on the constant in (1.7).

Proof. We shall use the following inequalities (see [3, Ch. 5, Sect. 11])

$$
\begin{aligned}
& \int_{0}^{T}\left|A_{\tilde{k}_{s}, i}^{+}(t)\right| d t \leq \gamma_{0} \frac{2^{\sigma \delta}}{k^{\delta}} \int_{\Omega_{T}}\left(u_{i}-k_{s}\right)_{+}^{\delta} d x d t \\
& \int_{\Omega_{T}}\left(u_{i}-\tilde{k}_{s}\right)_{+}^{\sigma} d x d t \leq \gamma_{0} \frac{2^{s(\delta-\sigma)}}{k^{\delta-\sigma}} \int_{\Omega_{T}}\left(u_{i}-k_{s}\right)_{+}^{\delta} d x d t, \quad \sigma<\delta<q,
\end{aligned}
$$

where $\gamma_{0}$ depends on the constant in (1.8). Setting $\bar{k}=\tilde{k}_{s}$ in (3.6) and using (3.20) we obtain

$$
\begin{aligned}
& \sum_{i=1}^{m}\left[\operatorname{ess} \sup _{t \in[0, T]} \int_{\Omega}\left(u_{i}-\tilde{k}_{s}\right)_{+}^{2} d x+2 \underline{\alpha} \int_{\Omega_{T}}\left|\nabla\left(u_{i}-\tilde{k}_{s}\right)_{+}\right|^{p} d x d t\right. \\
& \left.+2 \underline{\beta} \int_{\Omega_{T}}\left(u_{i}-\tilde{k}_{s}\right)_{+}^{p_{0}} d x d t\right] \leq C\left[\frac{2^{s(\delta-p)}}{k^{\delta-p}} Y_{s}+\sum_{j=1}^{2}\left(\frac{2^{s\left(\delta-\sigma_{j}\right)}}{k^{\delta-\sigma_{j}}} Y_{s}\right)^{1 / \sigma_{j}}\right. \\
& \left.+\frac{2^{s(\delta-1)}}{k^{\delta-1}} Y_{s}+\sum_{j=1}^{4}\left(\frac{2^{s \delta}}{k^{\delta}} Y_{s}\right)^{1-\gamma_{j}}\right] \equiv R
\end{aligned}
$$

where $C$ denotes here and in subsequent inequalities a positive constant depending on the data. Let

$$
\begin{aligned}
& a^{*}=\max \left\{\delta-p, \frac{\delta-\sigma_{1}}{\sigma_{1}}, \frac{\delta-\sigma_{2}}{\sigma_{2}}, \delta-1,\right. \\
&\left.\delta\left(1-\gamma_{1}\right), \delta\left(1-\gamma_{2}\right), \delta\left(1-\gamma_{3}\right), \delta\left(1-\gamma_{4}\right)\right\} \\
& a_{*}=\min \left\{\delta-p, \frac{\delta-\sigma_{1}}{\sigma_{1}}, \frac{\delta-\sigma_{2}}{\sigma_{2}}, \delta-1,\right. \\
&\left.\delta\left(1-\gamma_{1}\right), \delta\left(1-\gamma_{2}\right), \delta\left(1-\gamma_{3}\right), \delta\left(1-\gamma_{4}\right)\right\} \\
& \gamma_{*}=\max \left\{\frac{\mu}{p_{*}}, \frac{\nu}{p}, \gamma_{1}, \gamma_{2}, \gamma_{3}, \gamma_{4}\right\}
\end{aligned}
$$

and let $k>1$. Then from (3.21) using also (2.1) and (1.8) we obtain

$$
R \leq C \frac{2^{s a^{*}}}{k^{a_{*}}} Y_{s}^{1-\gamma_{*}}
$$


The functions

$$
w_{i}(x, t)=\left(u_{i}(x, t)-k_{s+1}\right)_{+}-\int_{\Omega}\left(u_{i}(x, t)-k_{s+1}\right)_{+} d x
$$

have zero averages in $\Omega$, thus, they satisfy the multiplicative inequality (1.7). Therefore we have

$$
\begin{aligned}
& \int_{\Omega_{T}}\left(u_{i}-k_{s+1}\right)_{+}^{q} d x d t \leq C \int_{\Omega_{T}}\left|\nabla\left(u_{i}-k_{s+1}\right)_{+}\right|^{p} d x d t \\
& \times\left(\operatorname{ess} \sup _{t \in[0, T]} \int_{\Omega}\left(u_{i}-k_{s+1}\right)_{+}^{2} d x\right)^{p / n} \\
& +|\Omega|^{1-q} \int_{0}^{T}\left(\int_{\Omega}\left(u_{i}-k_{s+1}\right)_{+} d x\right)^{q} d t .
\end{aligned}
$$

To estimate the last integral on the r.h.s. of (3.24) we consider

$$
\begin{aligned}
& \int_{\Omega}\left(u_{i}-k_{s+1}\right)_{+} d x \leq C \frac{2^{s}}{k} \int_{\Omega}\left(u_{i}-\tilde{k}_{s}\right)_{+}^{2} d x \\
& \leq C \frac{2^{s}}{k} \operatorname{ess} \sup _{t \in[0, T]} \int_{\Omega}\left(u_{i}-\tilde{k}_{s}\right)_{+}^{2} d x .
\end{aligned}
$$

Then by Hölder's inquality

$$
\begin{aligned}
& \left(\int_{\Omega}\left(u_{i}-k_{s+1}\right)_{+} d x\right)^{q} \leq\left(\int_{\Omega}\left(u_{i}-k_{s+1}\right)_{+} d x\right)^{q-1} \int_{\Omega}\left(u_{i}-k_{s+1}\right)_{+} d x \\
& \leq C \frac{2^{s}|\Omega|^{(q-1) / 2}}{k}\left(\int_{\Omega}\left(u_{i}-\tilde{k}_{s}\right)_{+}^{2} d x\right)^{\frac{(q-1)}{/} 2} \text { ess } \sup _{t \in[0, T]} \int_{\Omega}\left(u_{i}-\tilde{k}_{s}\right)_{+}^{2} d x \\
& \leq C \frac{2^{s}|\Omega|^{(q-1) / 2}}{k}\left(\operatorname{ess} \sup _{t \in[0, T]} \int_{\Omega}\left(u_{i}-\tilde{k}_{s}\right)_{+}^{2} d x\right)^{(q+1) / 2} .
\end{aligned}
$$

Hence

$$
\begin{aligned}
& |\Omega|^{1-q} \int_{0}^{T}\left(\int_{\Omega}\left(u_{i}-k_{s+1}\right)_{+} d x\right)^{q} d t \\
& \leq C \frac{2^{s}}{k} \frac{T}{|\Omega|^{(q+1) / 2}}\left(\operatorname{ess} \sup _{t \in(0, T)} \int_{\Omega}\left(u_{i}-\tilde{k}_{s}\right)_{+}^{2} d x\right)^{(q+1) / 2} .
\end{aligned}
$$

Combining (3.21)-(3.25) we obtain

$$
\int_{\Omega_{T}}\left(u_{i}-k_{s+1}\right)_{+}^{q} d x d t \leq C \frac{2^{s}}{k}\left[\left(\frac{2^{s a^{*}}}{k^{a_{*}}} Y_{s}^{1-\gamma_{*}}\right)^{(n+p) / n}\right.
$$




$$
\left.+\left(\frac{2^{s a^{*}}}{k^{a_{*}}} Y_{s}^{1-\gamma_{*}}\right)^{(q+1) / 2}\right] \leq C \frac{2^{s}}{k} \frac{2^{s a^{*}(n+p) / n}}{k^{a_{*}(n+p) / n}} Y_{s}^{\left(1-\gamma_{*}\right)(n+p) / n},
$$

where we have used the inequality $(q+1) / 2>(n+p) / n$. From $(3.20)$ it follows

$$
\begin{aligned}
& Y_{s+1} \equiv \sum_{i=1}^{m} \int_{\Omega_{T}}\left(u_{i}-k_{s+1}\right)_{+}^{\delta} d x d t \\
& \leq \sum_{i=1}^{m}\left(\int_{\Omega_{T}}\left(u_{i}-k_{s+1}\right)_{+}^{q} d x d t\right)^{\delta / q}\left(\int_{0}^{T}\left|A_{\tilde{k}_{s}, i}^{+}(t)\right| d t\right)^{1-\delta / q} \\
& \leq C \sum_{i=1}^{m}\left(\int_{\Omega_{T}}\left(u_{i}-k_{s+1}\right)_{+}^{q} d x d t\right)^{\delta / q}\left(\frac{2^{\delta s}}{k^{\delta}} Y_{s}\right)^{1-\delta / q} .
\end{aligned}
$$

From (3.26) and (3.27) we obtain (3.19). This concludes the proof.

Finally we arrive at

Theorem 3.3. Assume that $u_{0} \in L^{\infty}(\Omega)$ and

$$
\gamma_{*}<\frac{p}{n+p}
$$

then the solutions of problem $(\mathbf{P 1})$ and $(\mathbf{P} 2)$ are bounded and

$$
\sum_{i=1}^{m}\left\|u_{i}\right\|_{L^{\infty}\left(\Omega_{T}\right)} \leq \sum_{i=1}^{m}\left\|u_{0 i}\right\|_{l^{\infty}\left(\Omega_{T}\right)}+k_{*},
$$

where $k_{*}$ satisfies $(3.31)$.

Proof. To prove the theorem we apply either [2, 3, Lemma 4.1, Ch. 1] or [8, Lemma 5.6, Ch. 2], so we have to check whether $\alpha>0$ (see (3.19)). From (3.19) we obtain $\alpha=[(n+p) / n]\left[p /(n+p)-\gamma_{*}\right] \delta / q$, so $\alpha>0$ if $(3.28)$ holds.

Moreover, from these lemmas it follows that $Y_{s} \rightarrow 0$ as $s \rightarrow \infty$ if

$$
Y_{0} \leq\left(\frac{c_{*}}{k^{b_{*}}}\right)^{-1 / \alpha} 2^{-a^{*}\left(1 / \alpha^{2}\right)} .
$$

Since

$$
Y_{0} \leq \sum_{i=1}^{m} \int_{\Omega_{T}}\left(u_{i}-k_{0}\right)_{+}^{\delta} d x d t \leq C_{0},
$$

where $C_{0}$ is a positive constant dependeing on constants in the energy estimate (2.1) and in (1.7) we conclude that (3.30) holds if

$$
k \leq\left(c_{0} 2^{a^{*} / \alpha^{2}} c_{*}^{1 / \alpha}\right)^{\alpha / b_{*}} .
$$


This completes the proof.

Finally, we express more explicitely condition (3.28).

Proposition 3.4. The condition (3.28) is satisfied if the following conditions hold:

$$
\begin{aligned}
& 0<b<\frac{p^{2}}{p+n}-1 \quad \text { or } b=0 \\
& \mu<p^{*} \frac{p}{p+n} \\
& \nu<\frac{p^{2}}{p+n}
\end{aligned}
$$

and if $n<p^{2}-2 p$ then

$$
\begin{aligned}
& r_{1}<p^{*} \frac{p^{2}-2 p-n}{p(p+n)} \\
& r_{2}<\frac{p^{2}-2 p-n}{p+n}
\end{aligned}
$$

otherwise $a_{i j}=0$ for $i \neq j$.

\section{References}

[1] Alt, H.W., Luckhaus, S., Quasilinear elliptic-parabolic differential equations, Math. Z. 183 (1983), 311-341.

[2] DiBenedetto, E., Degenerate Parabolic Equations, Springer-Verlag, New York, 1993.

[3] DiBenedetto, E., Topics inquasilinear degenerate and singular parabolic equations, Bonn, Preprint 20, 1991.

[4] DeGiorgi, E., Sulla differenziabilita e l'analiticita delle estremali degli integrali multipli regolari, Mem. Accad. Sci. Torino Cl. Sci. Fis. Mat. Nat. 3 (1957), 25-43.

[5] Filo, J., Kačur, J., Local existence of general nonlinear parabolic systems, Nonlinear Anal. 24 (1995), 1597-1618.

[6] Gajewski, H., Gröger, K., Zacharias, K., Nichtlineare Operatorgleichungen und Operatordifferentielglaichungen, Akademie-Verlag, Berlin, 1974.

[7] Kurzweil, J., Ordinary Differential Equations, Elsevier Science Publishers, Amsterdam, 1986.

[8] Ladyzhenskaya, O.A., Solonnikov, V.A., Uraltseva, N.N., Linear and Quasilinear Equations of Parabolic Type, Amer. Math. Soc., Providence, RI, 1968.

[9] Zadrzyńska, E., Zajączkowski, W.M., On existence of solutions of mixed problems for parabolic systems, Topol. Methods Nonlinear Anal. 2 (1993), 125-145.

[10] Zajączkowski, W.M., $L_{\infty}$-estimate for solutions of nonlinear parabolic systems, Banach Center Publications 33, Warsaw, 1996. 
DARIUSZ WRZOSEK

Institute of ApPlied Mathematics

UNIVERSITY OF WARSAW

BANACHA 2

02-097 WarszaWA, Poland
WOJCIECH M. ZAJA̧CZKOWSKI

Institute of MATHEMATiCs Polish ACADEMY of ScIEnCES

ŚNIADECKICH 8 00-950 WarszaWA, Poland 